DOI: 10.12731/2227-930X-2016-1-27-37

\title{
ANALYTICAL SYNTHESIS OF CHEMICAL REACTOR CONTROL SYSTEM
}

\section{Labutin A., Nevinitsyn $V$.}

The problem of the analytical synthesis of the synergetic control system of chemical reactor for the realization of a complex series-parallel exothermal reaction has been solved. The synthesis of control principles is performed using the analytical design method of aggregated regulators. Synthesized nonlinear control system solves the problem of stabilization of the concentration of target component at the exit of reactor and also enables one to automatically transfer to new production using the equipment.

Keywords: analytical synthesis; control system; chemical reactor; synergetic control theory; computer simulation.

\section{Introduction}

The fundamental concept of the design of contemporary flow processes is the concept of the design of cybernetically organized chemical engineering processes and systems, which was rationalized by Academician V.V. Kafarov [1].

According to this concept, during the step of the design of chemical production, which is related to the conversion of initial substances to final products, the problem of optimal synthesis of reactor junction and problem of synthesis of the process control algorithms is solved; and, at the step of consumption, the sub-problem of the organization of optimal functioning of object under the effect of parametrical and signal disturbances [1-5].

Despite the large number of the works related to the automation and control of chemical reactors [6-9], the problem of synthesizing 
control systems that provide the maintenance of optimal modes of their work remains completely unsolved. This is related to the principal feature of chemical reactors as control objects, namely, manifold, nonlinearity, and multicoupling.

The solution of this situation is to develop a physical theory of control, in particular synergetic control theory, the principal features of which were formulated in [10-12].

The use of synergism ideas in the problems of control assumes the development and realization of the directed target self-organization of object-regulator dissipative nonlinear systems. Furthermore, the aim of the motion of system is formulated as the desired invariant manifold in phase space of object, which acts as a target attractor [12].

In general, the problem of synergetic synthesis of the control system is formulated as follows: the control principle, $u=\left(u_{1}, \ldots, u_{m}\right)^{\mathrm{T}}$, should be determined as the function of state variables of object $u_{1}\left(x_{1}, \ldots, x_{n}\right)$, $\ldots, u_{m}\left(x_{1}, \ldots, x_{n}\right)$, which transforms the representative point (RP) of system in phase space from the random initial state to the environment of the given invariant manifolds $\Psi_{S}\left(x_{1}, \ldots, x_{n}\right)=0, S=1, \ldots, m$ and subsequent motion along the intersection of manifolds to somewhat stationary point or to somewhat dynamic mode. In the given equations, $n$ is the dimensionality of state vector and $m$ is the number of external controls. On the path of motion, the minimum of the criterion of optimality of system $(\mathrm{J})$ should be attained and its stability should be ensured as follows:

$$
\mathbf{J}=\int_{0}^{\infty}\left[\sum_{S=1}^{m}\left(T_{S}^{2} \dot{\psi}_{S}^{2}+\psi_{S}^{2}\right)\right] \mathrm{d} \tau .
$$

The motion of RP in phase space follows the functional equation

$$
T_{S} \dot{\psi}_{S}+\psi_{S}=0, S=1, \ldots, m,
$$

where $T_{S}$ is time constant. This is the equation of stable critical point, which gives minimum to the optimizing functional (1). The condition of asymptotic stability of system generally has the form $T_{S}>0$. 
The effectiveness of the method of analytical design of control algorithms by nonlinear objects with the use of synergetic principle (the method of analytical design of aggregated regulators (ADAR)) is given in [13-16].

In this work, the problem of synthesizing the effective control algorithms of the chemical reactor in the realization of the complex series-parallel reaction is stated. The synthesized control system should provide the stabilization of the concentration of target component of chemical reaction on the exit of device under the action of disturbances on object, as well as the transfer of object from one mode of work to another (switch), namely, change of its productivity with the retention of the required quality of target component.

\section{Description of object and statement of control problem}

A chemical reactor is a capacity-type device equipped with a mechanical stirrer (Fig. 1). The device functions in isothermal mode. The multistep series-parallel reaction is carried out in the reactor as follows:

$$
\mathrm{A}+\mathrm{B} \stackrel{k_{1}}{\longrightarrow} \mathrm{P}_{1}, \mathrm{~A}+\mathrm{P}_{1} \stackrel{k_{2}}{\longrightarrow} \mathrm{P}_{2}, \mathrm{~A}+\mathrm{P}_{2} \stackrel{k_{3}}{\longrightarrow} \mathrm{P}_{3},
$$

where $\mathrm{A}$ and $\mathrm{B}$ are initial reagents; $\mathrm{P}_{1}, \mathrm{P}_{2}$ and $\mathrm{P}_{3}$ are products of reaction; $k_{1}, k_{2}$, and are rate constants of steps. The key component is $\mathrm{P}_{2}$ substance. Initial reagents $\mathrm{A}$ and $\mathrm{B}$ are given to the device by separate flows.

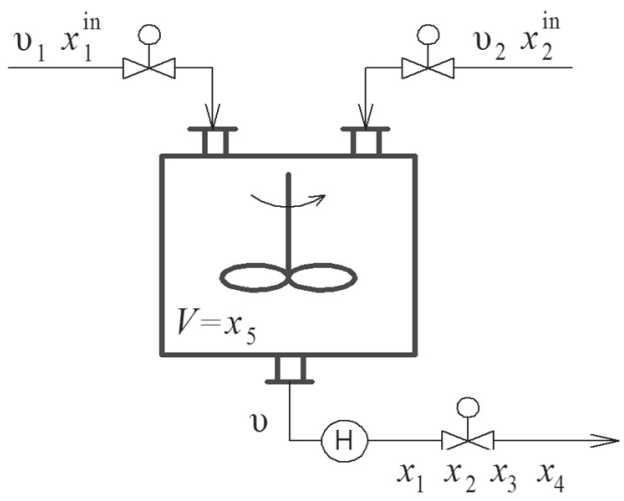

Fig. 1. Flow scheme of chemical reactor 
In Fig. 1, the following designations are given: $x_{1}^{\text {in }}, x_{2}^{\text {in }}$ are the concentrations of initial reagents; $v_{1}, v_{2}$ is the use of initial reagents; $v$ is the use of reaction mixture on the exit from device; $x_{1}, x_{2}, x_{3}$, and $x_{4}$ are the concentrations of components $\mathrm{A}, \mathrm{B}, \mathrm{P}_{1}$, and $\mathrm{P}_{2}$ in reactor; and $V=x_{5}$ is the volume of reaction mixture in device.

The mathematical model of chemical reactor at constant temperature of reaction mixture and variable degree (volume) has the following form:

$$
\begin{gathered}
\frac{\mathrm{d} x_{1}}{\mathrm{~d} \tau}=R_{1}+\frac{v_{1} x_{1}^{\text {in }}}{x_{5}}-\frac{v x_{1}}{x_{5}}, \frac{\mathrm{d} x_{2}}{\mathrm{~d} \tau}=R_{2}+\frac{v_{2} x_{2}^{\text {in }}}{x_{5}}-\frac{v x_{2}}{x_{5}}, \frac{\mathrm{d} x_{3}}{\mathrm{~d} \tau}=R_{3}-\frac{v x_{3}}{x_{5}}, \\
\frac{\mathrm{d} x_{4}}{\mathrm{~d} \tau}=R_{4}-\frac{v x_{4}}{x_{5}}, \frac{\mathrm{d} x_{5}}{\mathrm{~d} \tau}=v_{1}+v_{2}-v,
\end{gathered}
$$

where $R_{1}=-k_{1} x_{1} x_{2}-k_{2} x_{1} x_{3}-k_{3} x_{1} x_{4}, R_{2}=-k_{1} x_{1} x_{2}, R_{3}=k_{1} x_{1} x_{2}-k_{2} x_{1} x_{3}$, $R_{4}=k_{2} x_{1} x_{3}-k_{3} x_{1} x_{4}$ is the rate of reaction on components.

The problem of control of chemical reactor is formulated as follows: one should synthesize the control principle, which provides the transfer of device from one production, $G=\bar{x}_{4} \mathrm{v}$, to new production, $\bar{G}=\bar{x}_{4} \bar{v}$, and stabilization of the concentration of target component at the given degree $\bar{x}_{4}$ under the action of disturbances. The change of the output flow with time from $v$ to $\bar{v}$ can proceed by any principle, including in a stepwise manner.

The transfer to new production $\bar{G}$, with the maintenance of the given concentration $\bar{x}_{4}$, is made possible by the selection of the particular value of the average time of reaction mixture in device, $\bar{\tau}$. At the given $\bar{G}, \bar{x}_{4}, \bar{v}$, the required mean time can be achieved by the change in the mixture volume in the device.

The flow of the initial reagent $v_{2}$ at the input to the device is suggested as the control effect for the volume regulation. In addition, one should also choose the control for stabilizing the concentration $x_{4}$ at the given degree under the action of disturbances. The analysis of the structure of equations of mathematical model of reactor (3) shows that 
variables $x_{1}$ and $x_{3}$ may act as the internal controls and the direct external effect can be performed only on $x_{1}$ by the change of the consumption of initial reagent $v_{1}$ at the input to reactor. Thus, the control channels of the concentration of the target component and volume of the mixture in the device are represented as follows: $u_{1} \rightarrow x_{1} \rightarrow x_{4}, u_{2} \rightarrow x_{5}$, where $u_{1}=x_{1}, u_{2}=x_{2}$.

\section{Synthesis of control principles via ADAR}

Because the mathematical model of object (3) contains two external controlling effects $u_{1}=v_{1}$ and $u_{2}=v_{2}$, we use the ADAR method on the basis of parallel-series combination of invariant manifolds [12]. The procedure for synthesizing the control principle involves the following. At the first step, the invariant manifolds are considered as shown below:

$$
\psi_{S}\left(x_{1}, \ldots, x_{5}\right)=0, S=1,2,
$$

which determines the given relationships between phase coordinates of object, which in turn reflects the specificity of control object and requirements of designer to system. The control principle $u=\left(u_{1}, u_{2}\right)^{\mathrm{T}}$ is synthesized so as to perform the transition of representative point of system in phase space from arbitrary initial position to the intersection of manifolds, $\psi_{1,2}\left(x_{1}, \ldots, x_{5}\right)=0$.

Let us introduce two aggregated macrovariables to consideration, the first of which determines the relationship of $x_{1}$ with controlled variable $x_{4}$ and the second reflects the technological requirement to the volume of reaction system as follows:

$$
\psi_{1}=x_{1}+v_{1}\left(x_{4}\right), \psi_{2}=x_{5}-\bar{x}_{5},
$$

where $v_{1}\left(x_{4}\right)$ is somewhat function, which should be determined at subsequent procedure of synthesis. Macrovariables (4) should follow the solution of principal functional equation of ADAR method (2).

Let us introduce the macrovariables $\Psi_{1}$ and $\Psi_{2}$ of Eq. (4) to functional equation (2) for the synthesis of control principle, $u=\left(u_{1}, u_{2}\right)^{\mathrm{T}}$. As a result, we obtain the following equations: 


$$
T_{1}\left[\frac{\mathrm{d} x_{1}}{\mathrm{~d} \tau}+\frac{\partial v_{1}}{\partial x_{4}} \frac{\mathrm{d} x_{4}}{\mathrm{~d} \tau}\right]+x_{1}+v_{1}=0, T_{2} \frac{\mathrm{d} x_{5}}{\mathrm{~d} \tau}+x_{5}-\bar{x}_{5}=0 .
$$

Due to the equations of object (3), these relationships have the following form:

$$
\begin{gathered}
T_{1}\left[R_{1}+\frac{u_{1} x_{1}^{\mathrm{in}}}{x_{5}}-\frac{\bar{v} x_{1}}{x_{5}}+\frac{\partial v_{1}}{\partial x_{4}}\left(R_{4}-\frac{\bar{v} x_{4}}{x_{5}}\right)\right]+x_{1}+v_{1}=0, \\
T_{2}\left(u_{1}+u_{2}-\bar{v}\right)+x_{5}-\bar{x}_{5}=0,
\end{gathered}
$$

where $\bar{v}$ is the new set value of the consumption at the exit from reactor, which determines the required production.

We obtain the following relationships for the control principle from Eq. (5):

$$
\begin{gathered}
u_{1}=-\frac{\left(x_{1}+v_{1}\right) x_{5}}{T_{1} x_{1}^{\text {in }}}-\frac{R_{1} x_{5}}{x_{1}^{\text {in }}}+\frac{\bar{v} x_{1}}{x_{1}^{\text {in }}}-\frac{\partial v_{1}}{\partial x_{4}} \frac{\left(R_{4} x_{5}-x_{4} \bar{v}\right)}{x_{1}^{\text {in }}}, \\
u_{2}=-\frac{\left(x_{5}-\bar{x}_{5}\right)}{T_{2}}+\bar{v}-u_{1} .
\end{gathered}
$$

Controls $u_{1}$ and $u_{2}$ transfer the RP of the system in the phase space to the intersection of manifolds, $\Psi_{1}=0$ and $\Psi_{2}=0$, where the relationships $x_{1}=-v_{1}, x_{5}=\bar{x}_{5}$ are realized and the compression of phase space is realized, i.e., a decrease occurs in the dimensionality of the system of equations (3). The equations of decomposed system with the assumption of relationships $x_{1}=-v_{1}$ and $v=\bar{v}$ have the following form:

$$
\frac{\mathrm{d} x_{2}}{\mathrm{~d} \tau}=R_{2}+\frac{u_{2} x_{2}^{\text {in }}}{x_{5}}-\frac{\bar{v} x_{2}}{x_{5}}, \frac{\mathrm{d} x_{3}}{\mathrm{~d} \tau}=R_{3}-\frac{\bar{v} x_{3}}{x_{5}}, \frac{\mathrm{d} x_{4}}{\mathrm{~d} \tau}=R_{4}-\frac{\bar{v} x_{4}}{x_{5}},
$$

where $R_{2}=k_{1} v_{1} x_{2}, R_{3}=-k_{1} v_{1} x_{2}+k_{2} v_{1} x_{3}, R_{4}=-k_{2} v_{1} x_{3}+k_{3} v_{1} x_{4}$.

The function $v_{1}\left(x_{4}\right)$ in the decomposed system (7) can be considered to be the internal control, under the action of which the motion of object (7) along the intersection of manifolds $\Psi_{1,2}=0$ takes place. At the second step of procedure, the investigation of the expression for $v_{1}\left(x_{4}\right)$ is performed. For this purpose, the aim of the motion of system 
(7) is considered in the form of invariant manifold, which reflects the technological requirement to system as follows:

$$
\Psi_{3}=x_{3}-\bar{x}_{4}=0 \text {. }
$$

Macrovariable $\Psi_{3}$ corresponds to the solution of functional equation, $T_{3} \dot{\psi}_{3}+\psi_{3}=0$, which has the following form in extended form with the assumption of Eq. (8) due to the model of decomposed system (7) shown below:

$$
T_{3}\left(-k_{2} v_{1} x_{3}+k_{3} v_{1} x_{4}-\frac{\bar{v} x_{4}}{x_{5}}\right)+x_{4}-\bar{x}_{4}=0 .
$$

The internal control is written as follows in accordance with Eq. (9):

$$
v_{1}=\frac{x_{4}-\bar{x}_{4}}{T_{3}\left(k_{2} x_{3}-k_{3} x_{4}\right)}-\frac{x_{4} \bar{v}}{x_{5}\left(k_{2} x_{3}-k_{3} x_{4}\right)} .
$$

Final equation for the control principle $u_{1}$ can be obtained by the introduction of $v_{1}$ function (10) and its partial derivative $\partial v_{1} / \partial x_{4}$ to Eq. (6). The parameters of the adjustment of control laws, which affect the quality of the dynamics of the processes in object-regulator isolated system, are the time constants $T_{1}, T_{2}$ and $T_{3}$. The conditions of asymptotic stability have the following form: $T_{1}>0, T_{2}>0, T_{3}>0$.

\section{Mathematical simulation and results}

In order to verify the operation of the synthesized control law by the chemical reactor, the computer simulation of the object-regulator isolated system was performed. Properties of the control system, such as the ability (transfer) of the chemical reactor to switch from one mode of work to another (transfer to new production), disturbance invariance, covariance to the given actions, and the asymptotic stability of isolated system, were also studied.

The simulation was performed with the following technological and constructive parameters of object: $V=\bar{x}_{5}=500 \mathrm{~L}, x_{1}^{\text {in }}=19.74$ $\mathrm{mol} / \mathrm{L}, x_{2}^{\text {in }}=10.93 \mathrm{~mol} / \mathrm{L}, v_{1}=1.5 \mathrm{~L} / \mathrm{min}, v_{2}=3.5 \mathrm{~L} / \mathrm{min}, v=5 \mathrm{~L} /$ min, activation energy $E_{1}=60300 \mathrm{~J} / \mathrm{mol}$, preexponential multiplier of rate constant $k_{1} k_{10}=109860 \mathrm{~L} /(\mathrm{mol} \mathrm{min})$, ratios of rate constants of 
consecutive steps $k_{2} k_{1}=2, k_{3} k_{1}=2.5$, and the given concentration of target component $\bar{x}_{4}=0.54 \mathrm{~mol} / \mathrm{L}$. The parameters of adjustment of regulators are $T_{1}=20 \mathrm{~min}, T_{2}=50 \mathrm{~min}$, and $T_{3}=50 \mathrm{~min}$.

In Figs. 2-4, the examples of transient control processes in the object-regulator isolated system are given with the transition from production $G=\bar{x}_{4} \mathrm{v}=2.7 \mathrm{~mol} / \mathrm{min}$ to production $\bar{G}=\bar{x}_{4} \bar{v}=2.16 \mathrm{~mol} / \mathrm{min}$ by the change of stress. In Fig. 2, which demonstrates the change of regulated variables, the variant, when switching is performed in manual mode, is given.

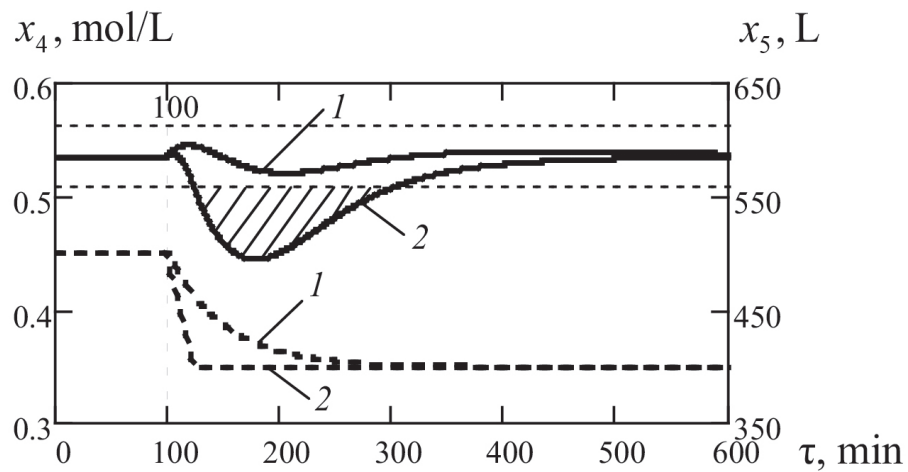

Fig. 2. Change of controlled variable $x_{4}$ (bold line) and the volume of mixture in device $x_{5}$ (dashed line) with decrease in load $v$ by $20 \%$ : (1) control system $u_{1}, \mathrm{~L} / \mathrm{min}$

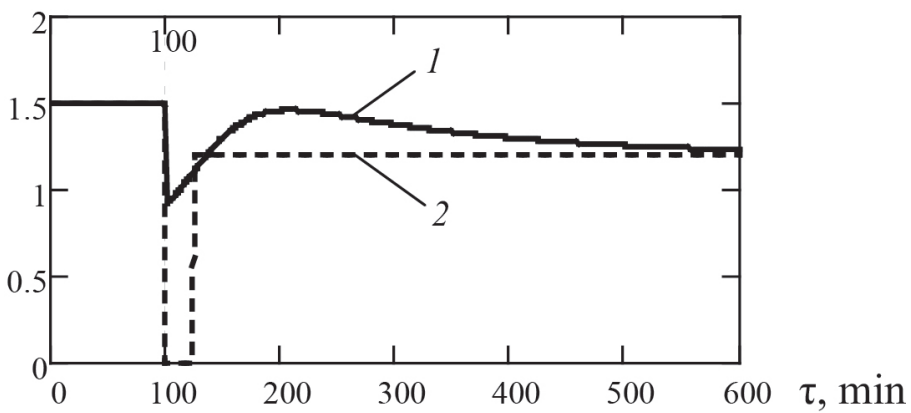

Fig. 3. Change in control action $u_{1}=v_{1}$ with decrease in load $v$ by $20 \%$ :

(1) control system and (2) manual mode 
$u_{2}, \mathrm{~L} / \mathrm{min}$

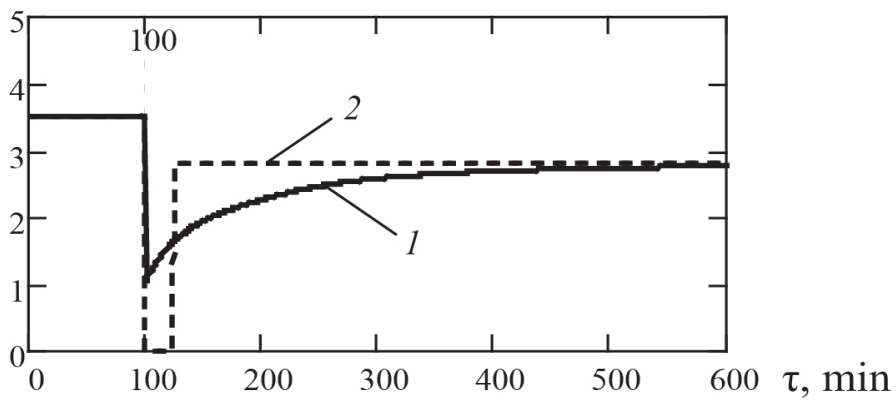

Fig. 4. Change in control action $u_{2}=v_{2}$ with decrease in load $v$ by $20 \%$ :

(1) control system and (2) manual mode

As follows from Fig. 2, the major drawback of the manual mode of transfer to new production is the large over-regulation on the concentration of target component, which leads to the loss of product.

\section{Conclusions}

In this work, the problem of the analytical synthesis of the control law of chemical reactor with the transition from one production to another was solved via the methods of synergetic theory. Computer simulation of the object-regulator isolated system confirmed these properties of synthesized control system as the ability to switch chemical reactor from one mode of work to another (transfer to new production), disturbance invariance, covariance to the given actions, and asymptotic stability. These facts make synergetic control theory very promising applied to such complex, manifold, and nonlinear objects of chemical engineering as chemical reactors.

\section{References}

1. Kafarov V.V. Metody kibernetiki v khimii i khimicheskoi tekhnologii [Cybernetic Methods in Chemistry and Chemical Engineering]. Moscow: Khimiya, 1985, 4th ed. 
2. Ostrovskii G.M.,Volin Yu.M. Metody optimizatsii khimicheskikh reaktorov [Chemical Reactor Optimization Methods]. Moscow: Khimiya, 1976.

3. Labutin A.N. Optimization of flexible multiproduct continuous reactor systems. Izv. Vyssh. Uchebn. Zaved., Khim. Khim. Tekhnol., 1999, vol. 42. P. 117.

4. Labutin A.N., Isaenkov A.E., Volkova G.V. Optimal synthesis of flexible reactor systems. Izv. Vyssh. Uchebn. Zaved., Khim. Khim. Tekhnol., 2010, vol. 53. P. 125.

5. Dvoretskii D.S., Dvoretskii S.I., Ostrovskii G.M. Integrated design of power- and resource-saving chemical processes and process control systems: strategy, methods, and application. Theor. Found. Chem. Eng., 2008, vol. 42. P. 26.

6. Avtomaticheskoe upravlenie v khimicheskoi promyshlennosti [Computer-Aided Manufacturing in the Chemical Industry]. Dudnikov, E.G., Ed., Moscow: Khimiya, 1987.

7. Bespalov A.V., Kharitonov N.I. Sistemy upravleniya khimiko-tekhnologicheskimi protsessami [Control Systems for Chemical Technology Processes]. Moscow: Akademkniga, 2007.

8. Sukharev A.V., Labutin A.N., Golovushkin B.A., Erofeeva E.V., Chemical reactor control issues. Izv. Vyssh. Uchebn. Zaved., Khim. Khim. Tekhnol., 2011, vol. 54. P. 119.

9. Lopez-Perez P.A., Neria-Gonzalez M.I., Aguilar-Lopez R. Nonlinear controller design with application to a continuous bioreactor. Theor. Found. Chem. Eng., 2013, vol. 47. P. 585.

10. Kol'tsova E.M., Gordeev L.S. Metody sinergetiki v khimii i khimicheskoi tekhnologii [Synergistic Methods in Chemistry and Chemical Engineering]. Moscow: Khimiya, 1999.

11. Krasovskii A.A. Development and formation of the contemporary theory of control, in Sinergetika i problemy teorii upravleniya [Synergetics and Problems of Control Theory], Kolesnikov A.A., Ed., Moscow: Fizmatlit, 2004. 
12. Kolesnikov A.A., Sinergeticheskaya teoriya upravleniya [Synergistic Theory of Control]. Moscow: Energoatomizdat, 1994.

13. Labutin A.N., Nevinitsyn V.Yu., Volkova G.V. Synthesis and modeling of a multidimensional control system of a chemical reactors cascade. Izv. Vyssh. Uchebn. Zaved., Ser. Ekon., Finansy Upravl. Proizvodstvom, 2012, no. 2. P. 150.

14. Labutin A.N., Kukushkin A.V. Synergistic approach to reactor temperature control. Izv. Vyssh. Uchebn. Zaved., Ser. Ekon., Finansy Upravl. Proizvodstvom, 2011, no. 3. P. 81.

15. Labutin A.N., Nevinitsyn V.Yu. Synergistic synthesis of concentration regulator for the target product in a cascade of chemical reactors. $I z v$. Vyssh. Uchebn. Zaved., Ser. Ekon., Finansy Upravl. Proizvodstvom, 2011, no. 3. P. 86.

16. Labutin A.N., Nevinitsyn V.Yu. Synergistic synthesis of a chemical reactor control system. Izv. Vyssh. Uchebn. Zaved., Ser. Khim. Khim. Tekhnol., 2012, vol. 55. P. 104.

\section{DATA ABOUT THE AUTHORS \\ Labutin Alexander \\ Ivanovo State University of Chemistry and Technology \\ 7, Sheremetevskiy Avenue, Ivanovo, 153000, Russian Fede- ration}

\section{Nevinitsyn Vladimir}

Ivanovo State University of Chemistry and Technology

7, Sheremetevskiy Avenue, Ivanovo, 153000, Russian Federation

nevinitsyn@gmail.com 